\title{
Propagation of nitrogen gas in a liquid helium cooled vacuum tube following sudden vacuum loss- Part II: Analysis of propagation speed
}

\author{
R. C. Dhuley ${ }^{1,2}$ and S. W. Van Sciver ${ }^{1,2}$ \\ ${ }^{1}$ National High Magnetic Field Laboratory, Tallahassee, FL 32310 \\ ${ }^{2}$ Mechanical Engineering Department, FAMU-FSU College of Engineering, Tallahassee, FL 32310
}

\begin{abstract}
The propagation of near-atmospheric nitrogen gas entering a liquid helium (LHe) cooled vacuum tube following an accidental loss of vacuum will be strongly influenced by condensation of the gas on the tube wall. Our previous experimental study revealed that in presence of condensation, the propagation speed of the gas front decreases nearly exponentially as the front advances in the tube. In the present paper the exponential decrease is studied analytically. We reduce the analytical model of the front speed, using assumptions, to show its derivative in the direction of propagation to be proportional to the mass deposition rate near the front. The deposition rate is then calculated at discreet locations along the tube from the condensation heat transfer rate at these locations. We find the deposition rate to diminish nearly exponentially along the tube so that the spatial derivative of the speed will show the same effect. In this special case the front speed will also fall exponentially along the tube. Within the experimental and procedural uncertainty, the exponential decay coefficient of the mass deposition rate also agrees reasonably with the empirically determined exponential decay coefficient of the propagation speed.
\end{abstract}

Keywords: Condensation, air propagation, loss of vacuum, particle accelerator, liquid helium

\section{Introduction}

The beam-line of a superconducting particle accelerator is a long channel holding high vacuum on its inside while being immersed in liquid helium (LHe) [1]. If this beam-line accidentally ruptures at a location exposed to atmospheric air, the air will rapidly flow into the channel and propagate down the vacuum space. The propagation behavior of the air front must be known in order to arrest the front and restrict the beam-line contamination to a minimal length. Past work on this subject has shown the loss-ofvacuum induced air propagation in a beam-line [2] or in a simple LHe cooled tube [3] to be substantially slower than in a vacuum tube at room temperature [4]. By systematically studying propagation of nitrogen gas (a substitute of air) in high vacuum tubes at different wall temperatures, our previous report [5] identified gas condensation to be a must for this slow propagation. Our experiments also brought out an interesting feature that the front in presence of condensation gets slower and slower as it advances in the channel. Additionally, the empirical fits to the measured propagation data showed the front speed along the channel to decrease nearly exponentially. An analytical model formulated for the front propagation speed explained why the speed decreases along the tube, but did not provide any insight as to why this decrease is exponential.

The analyses presented in this paper provide further theoretical and experimental support to the previously observed exponential fall in the propagation speed. We reduce the analytical expression of the 
propagation speed to a form that can be compared directly with the empirical fits to our experimental data. The parameters required to work with this form are determined from the calculated rates of condensation heat transfer and mass deposition on the tube. The spatial variation of these parameters along the tube is then studied. The result of this procedure shows that the propagation speed given by the analytical model also falls off exponentially along the tube with nearly the same exponential decay coefficient as that of the empirical fit.

\section{Experimental setup and procedure}

Elaborate details of the experimental setup and the experimental procedure are given in [6]. Figure 1 depicts the general characteristics of the experimental setup. In this setup a copper tube $(1.5 \mathrm{~m}$ long, $38 \mathrm{~mm}$ outer diameter, and $3 \mathrm{~mm}$ thick) evacuated to $\approx 10^{-4} \mathrm{~Pa}$ is immersed in a large bath of LHe at $4.2 \mathrm{~K}$. The tube vacuum is isolated from a tank containing nitrogen gas by means of a fast-opening solenoid valve (SV). On opening this valve the room-temperature nitrogen gas in the tank flows rapidly into the tube, propagates down the vacuum space, and condenses on the inner surface of the cold tube. The tube carries on its outer surface twelve thermometers equally spaced by $0.125 \mathrm{~m}$ along the length (except the last pair, which has $0.25 \mathrm{~m}$ spacing). These thermometers measure the rise in the tube temperature resulting from the condensation of the warm gas on the cold tube. The condensation heat and mass transfer calculations presented further in this paper essentially use the same temperature data that were previously used in determining the front propagation speed [5]. We recall here also from [5] that these temperature data have been obtained from three separate experiments that started with $50 \mathrm{kPa}$, $100 \mathrm{kPa}$, and $150 \mathrm{kPa}$ nitrogen gas in the supply tank. In this paper we have in general referred to the experiments as ' $50 \mathrm{kPa}$ experiment', ' $100 \mathrm{kPa}$ experiment', and ' $150 \mathrm{kPa}$ experiment' according to the

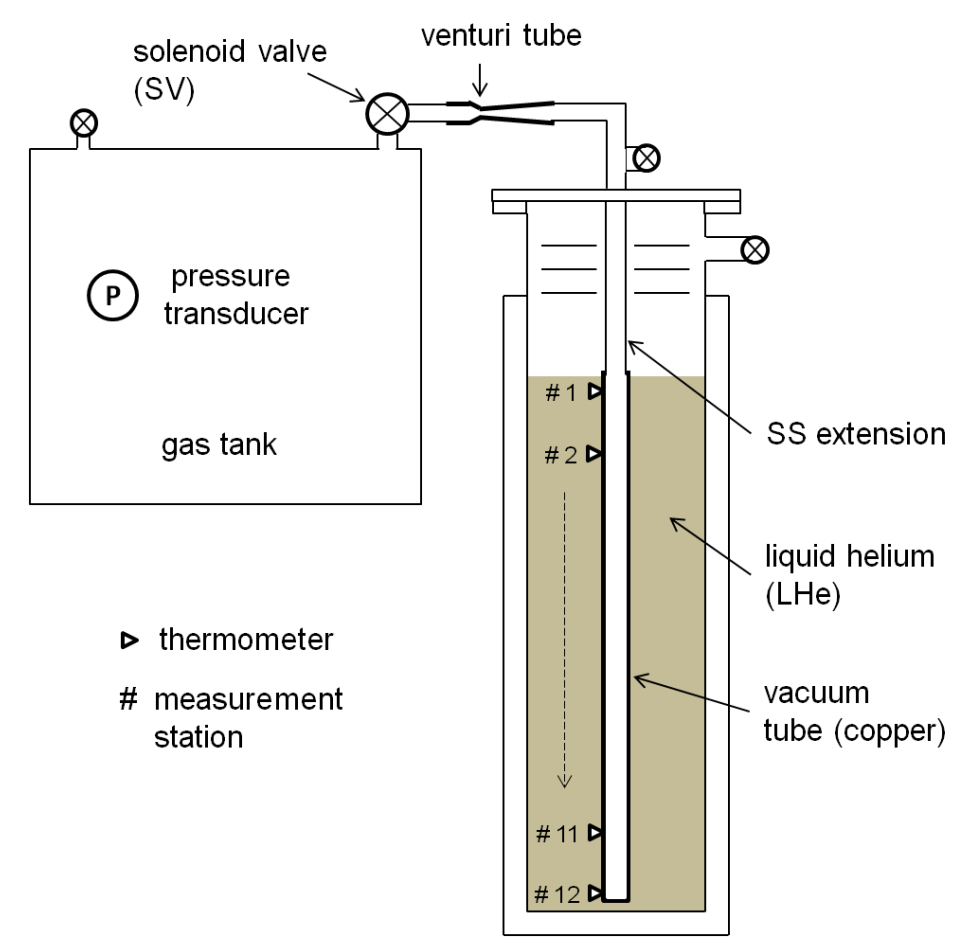

Figure 1: Schematic of the experimental setup used for studying the propagation of nitrogen gas in a LHe cooled vacuum tube. 
tank pressure at which that experiment started.

\section{Analytical modeling}

\section{a) Previous observations and modeling [5]}

The principal observation from our measurement of propagation speed is that the front speed decreases along the vacuum tube. This deceleration is consistent among the $50 \mathrm{kPa}, 100 \mathrm{kPa}$, and $150 \mathrm{kPa}$ experiments, which essentially had the gas vented to the vacuum tube at different mass-flow rates. Furthermore on regression analysis the front propagation speed in the vacuum tube is seen to follow an exponential decay relation of the form:

$$
\left.v\right|_{x}=(b / a) e^{-x / b}
$$

In this expression $\left.v\right|_{x}$ is the front speed when the front is located at $x$ in the tube (the tube entrance is at $x=0$ ), while $a$ and $b$ are parameters of the empirical fit to the measured propagation data. These parameters for the three experiments are summarized in Table 1:

Table 1: Parameters $a$ and $b$ appearing in

$\left.v\right|_{x}=(b / a) e^{-x / b}$ with their standard errors [5]

\begin{tabular}{ccc}
\hline $\mathrm{p}_{\text {start }}[\mathrm{kPa}]$ & $\mathrm{a}[\mathrm{s}]$ & $\mathrm{b}[\mathrm{m}]$ \\
\hline 50 & $0.041 \pm 0.005$ & $0.46 \pm 0.02$ \\
100 & $0.031 \pm 0.005$ & $0.63 \pm 0.04$ \\
150 & $0.035 \pm 0.010$ & $0.95 \pm 0.15$ \\
\hline
\end{tabular}

We also formulated an analytical expression for the front propagation speed by applying conservation of mass to the scenario depicted in figure 2. In the illustration of figure 2, the gas at an in-flow rate $\dot{m}_{i n}$ enters a cold vacuum tube of inner diameter $D$ at location $X=0$ and a gas front propagates along the $x$ direction. The local gas density is $\rho$ and the local rate of mass deposition (condensation) on the inner surface of the tube is $\dot{m}_{d e p}$. For this scenario the front speed when the front is located at $X=x$ is given by (conditions at the front are denoted by $\left.\right|_{x}$ ):

$$
\left.v\right|_{x}=\frac{\dot{m}_{i n}-\pi D \int_{0}^{x} \dot{m}_{d e p}(X) d X}{\left.\pi D^{2} \rho\right|_{x} / 4}
$$

As elaborated in [5], equation 2 accounts for the observed decrease in the propagation speed with increasing travel length $x$. We now further work with this model to show that the decrease with travel length is nearly exponential as our experimental data have pointed out. 


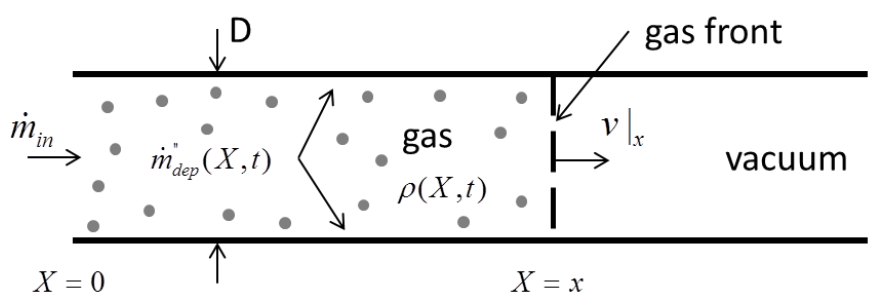

Figure 2: Propagation of a condensable gas in a vacuum tube.

\section{b) Extension of the analytical model}

Differentiating with $x$, the empirical relation $\left.v\right|_{x}=(b / a) e^{-x / b}$ leads to:

$$
\left.(d v / d x)\right|_{x}=(-1 / a) e^{-x / b}
$$

which implies that the spatial derivative of the propagation speed should also decay exponentially along the tube. Similarly, equation 2 on differentiation with $x$ gives:

$$
\left.(d v / d x)\right|_{x}=\frac{-\left.4 \dot{m}_{d e p}^{\prime \prime}\right|_{x}}{\left.D \rho\right|_{x}}
$$

In this derivation, we have assumed that the gas density at the front does not vary significantly along the tube so that $\left.(d \rho / d X)\right|_{x} \approx 0$. If conditions given by equations 3 and 4 are equivalent, then it follows that the rate of mass deposition at the front $\left.\dot{m}_{d e p}^{\prime \prime}\right|_{x}$ should decrease exponentially as the front advances along $x$ and should have the same exponential decay coefficient $b$ as in equation 3 . The mass deposition rate at the front when the front is located at $x$ should then take the form:

$$
\left.\dot{m}_{d e p}\right|_{x} \propto e^{-x / b}
$$

In our previous report, we discussed some inherent difficulties that do not allow calculating the propagation speed from its analytical form (equation 2). The main difficulty is quantifying the mass deposition rate over the extent of condensation, i.e., from $X=0$ to $X=x$ so as to evaluate the integral term in equation 2 . The procedure described above essentially circumvents this difficulty by removing this integral and working only with the mass deposition rate at the front. The local mass deposition rate can be obtained as shown further by calculating the condensation heat transfer rates and accounting for the enthalpy change associated with the condensation process.

\section{Condensation heat transfer}

The rate of heat transfer incident on the tube due to gas condensation is calculated using the tube temperature vs. time data obtained from experiments. Note that in this paper the term 'condensation' refers in general to the process of phase change, which in our experiments is de-sublimation or direct gas to solid conversion without encountering a liquid state. 


\section{a) The tube temperature data}

After venting the nitrogen gas in the supply tank to the LHe cooled vacuum tube, the gas condenses on the inner surface of the tube. A part of the heat incident on the tube due to this condensation transfers to the LHe contacting the tube outer surface, while the rest accumulates in the heat capacity of the tube wall. The two competing processes of heat addition (by gas condensation) and heat removal (by LHe) cause the tube temperature to evolve with time in a manner shown in figure 3. These maps present the temperature data obtained from the $50 \mathrm{kPa}$ experiment (figure 3(a)), $100 \mathrm{kPa}$ experiment (figure 3(b)), and $150 \mathrm{kPa}$

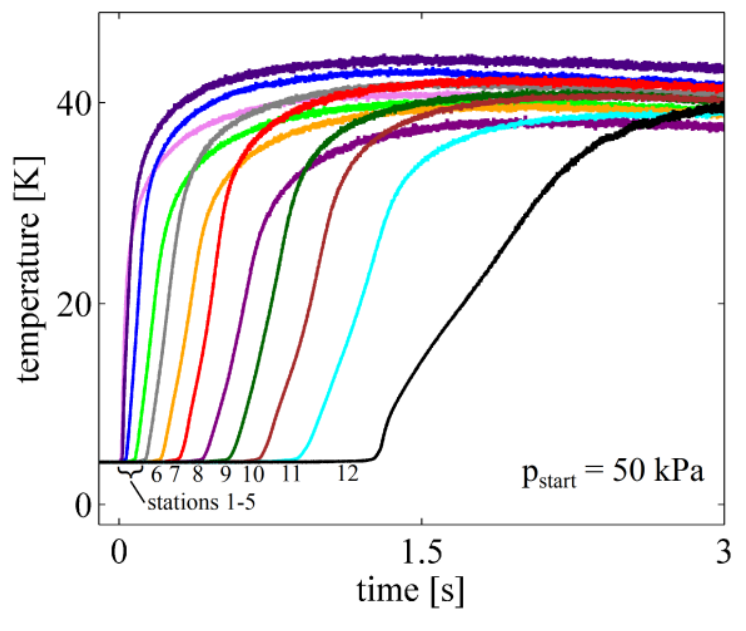

(a)

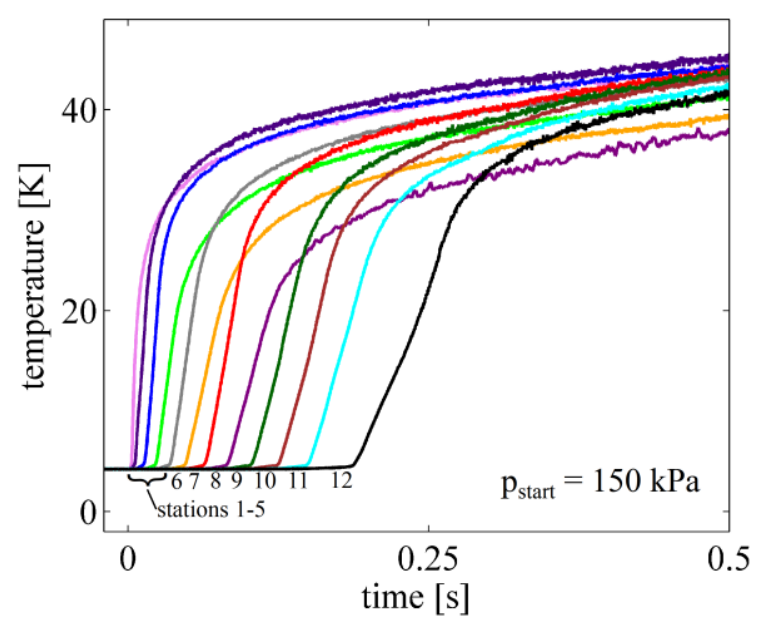

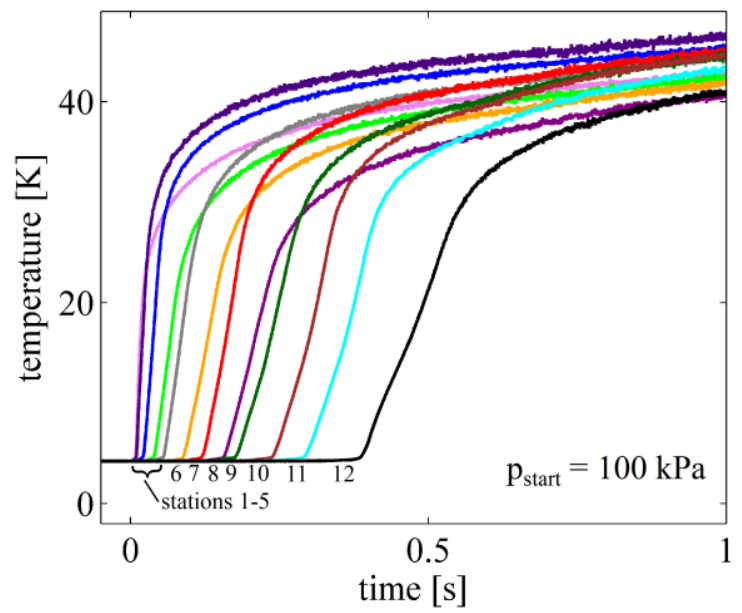

(b)

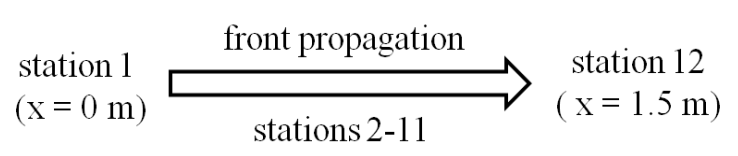

Color key

Trace Station Trace Station

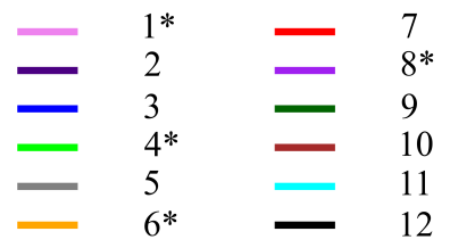

(c)

Figure 3: (Color online) Temperature rise with time due to gas condensation at the twelve thermometer stations on the tube during (a) $50 \mathrm{kPa}$ experiment (b) $100 \mathrm{kPa}$ experiment (c) $150 \mathrm{kPa}$ experiment. The gas front travels from station 1 at the entrance of the tube to station 12 near the rigid end. * in the Color key denotes stations with temperature measurement error. 
experiment (figure 3(c)). In each experiment the temperature was measured at twelve locations over the tube length and thus each plot in figure 3 has twelve temperature traces. The trace originating at time $t=0$ comes from the thermometer at the tube entrance (station 1) while the trace originating at the latest time comes from the far end of the tube (station 12). All other traces in between arise in order of the station location and highlight distinctly the propagation of the gas front from station 1 to station 12 .

The tube temperature at any station rises steeply after the onset of condensation at that station, followed by a 'knee' indicating a decrease in the rate of temperature rise, and finally proceeds towards a steady state. The steady state is apparent in figure 3(a) (50 kPa experiment) but takes longer to attain than the duration for which the data are plotted in figures 3(b) and 3(c) (100 kPa and $150 \mathrm{kPa}$ experiments). Note the discrepancy in the temperature traces of stations $1,4,6$, and 8- the initial temperature rise is less steep compared to the other eight traces and the knee appears at a lower temperature. We believe that the thermometers at these stations $(1,4,6$, and 8) fail to establish proper thermal contact with the tube and thus read a temperature lower than that actually exists at these stations. Repeated attempts to rectify this problem have not yielded much success.

The goal here is to obtain condensation heat transfer rates from the data of figure 3 . As stated earlier the heat incident due to condensation in part will accumulate in the tube wall and in part will transfer to LHe. The rate of heat accumulation can be obtained directly from the temperature traces. The heat transfer rate to LHe at a given time depends on the tube temperature at that time and on the prevailing LHe heat transfer regime. The LHe heat transfer regimes and other relevant characteristics of the temperature traces are identified in the following.

\section{b) Characteristics of the tube temperature traces}

Plotted in figure 4 is a sample temperature-time trace on a log-log scale. Although this sample trace is chosen arbitrarily to be the trace measured at station 5 during the $150 \mathrm{kPa}$ experiment, the characteristics

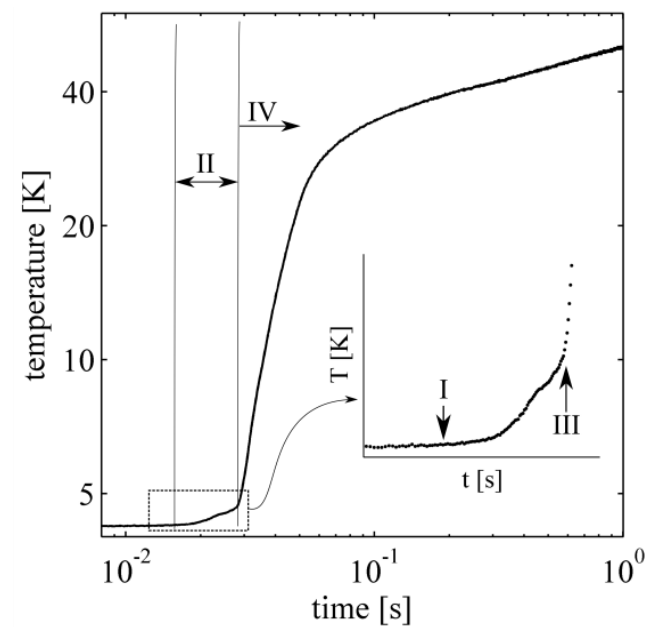

$150 \mathrm{kPa}$ experiment, Station 5

I: Onset of condensation

II: Convection/nucleate boiling in LHe

III: Onset of film boiling in LHe $(\mathrm{T} \approx 4.56 \mathrm{~K})$

IV: Unsteady film boiling in LHe

Figure 4: The tube temperature recorded at station 5 during the $150 \mathrm{kPa}$ experiment plotted with time on a $\log \log$ scale. The inset shows the trace for $\mathrm{T}<5 \mathrm{~K}$ in order to highlight the times of onset of condensation heat transfer (I) and LHe film boiling (III). Approximate duration of LHe nucleate boiling is the interval II, while IV represents unsteady LHe film boiling. 
of this trace are consistently seen among all the other traces (all stations, all experiments).

In figure 4, 'I' marks the onset of condensation at station 5. We assume that condensation at any station in the tube begins as soon as the gas arrives at this station so that the condensation onset time is the same as the gas front arrival time. The gas front arrival times at all stations have been obtained by the method described in our previous work [5]. Condensation at station 5 causes the tube to warm up and transfer heat to LHe from this station. The LHe heat transfer mode for few milliseconds after the onset is convection, typically when tube temperature is $<4.3 \mathrm{~K}$ [1]. The mode then transitions to nucleate boiling as the tube warms up above $4.3 \mathrm{~K}$. The convection/nucleate boiling regime is the region II in figure 4 . The heat transfer then transitions to film boiling as the tube further warms up due to the incident condensation. 'III' points to the onset of LHe film boiling, the time when the tube begins to suddenly warm up much faster than during nucleate boiling (see inset of figure 4). In all our experiments, film boiling is seen to start at a station when the temperature at that station exceeds $\approx 4.56 \mathrm{~K}$. Region IV in figure 4 represents unsteady film boiling in LHe at station 5. Note that in our experiments all of the LHe heat transfer modes (convection, nucleate boiling, and film boiling) are unsteady and so obtaining very accurate time dependent LHe heat transfer rates from just the measured temperature is not possible. The LHe heat transfer rates as determined in the following are therefore only approximate.

\section{c) Calculating the condensation heat transfer rate}

The condensation heat transfer rate $q_{d e p}$ at any location in the tube is given by the following heat balance relation (see Appendix for an illustration of the heat balance):

$$
q_{d e p}=\frac{O D^{2}-I D^{2}}{4 I D} \rho c \frac{d T}{d t}+\frac{O D}{I D} q_{L H e}
$$

In equation 6, ID and OD respectively are the inner and outer diameter of the tube, while $\rho$ and $c$ respectively are the density and specific heat capacity of the tube material (copper). The first term on RHS of equation 6 represents the rate of heat accumulation in the tube while $q_{L H e}$ denotes the heat transfer rate to LHe. Note that both the RHS terms in equation 6 depend only on the tube temperature and so the rate of condensation heat transfer can be evaluated at any location where a temperature measurement is available. Equation 6 thus allows calculating the condensation heat transfer rates at the twelve thermometer stations. The procedure to determine the local condensation heat transfer rates is only summarized here and is elaborated in Appendix. To calculate the rate of heat accumulation, the temperature dependent specific heat capacity and the rate of temperature rise are obtained from the temperature-time data. The LHe heat transfer rates are evaluated from the tube temperature using the Kutataladze correlation [1] in the nucleate boiling regime and Steward's data [7] in the film boiling regime. The calculated condensation heat transfer rates at the twelve stations for the $50 \mathrm{kPa}, 100 \mathrm{kPa}$, and $150 \mathrm{kPa}$ experiments are displayed in figure 5.

Each of the condensation heat transfer curve is seen to first steeply rise, attain a maximum, and then fall off to a near-steady value. This behavior can be qualitatively understood as follows. Recall that a front on passing a particular location will cause the pressure at that location to rise with time (see figure 3(a) of [5]). This rising pressure will cause the gas to condense on the tube at an increasing rate. As the rate of condensation heat transfer can be related to the rate of condensation mass transfer via the total enthalpy 
change during the process, we can infer that the rising pressure will cause the rate of condensation heat transfer to increase. This explains the rising phase of the condensation heat transfer traces. The increase in condensation heat transfer rate with pressure is, however, not indefinite. The heat incident on the tube due to condensation also raises the tube temperature and thereby reduces the capacity of the tube to further condense the gas. The result is a rapid decrease in the condensation mass and heat transfer rates, which is the falling phase of the traces in figure 5. Such behavior of gas condensation on cryogenic surfaces, although with gas-cryogen systems different than nitrogen-LHe, has been observed in previous research $[8,9,10]$ related to the development of cryogenic pumps. Studying this dynamics of

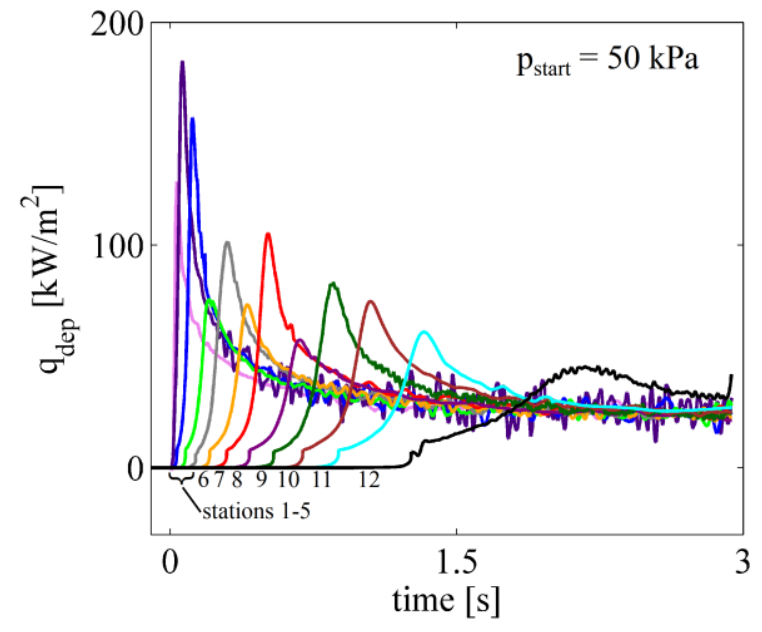

(a)

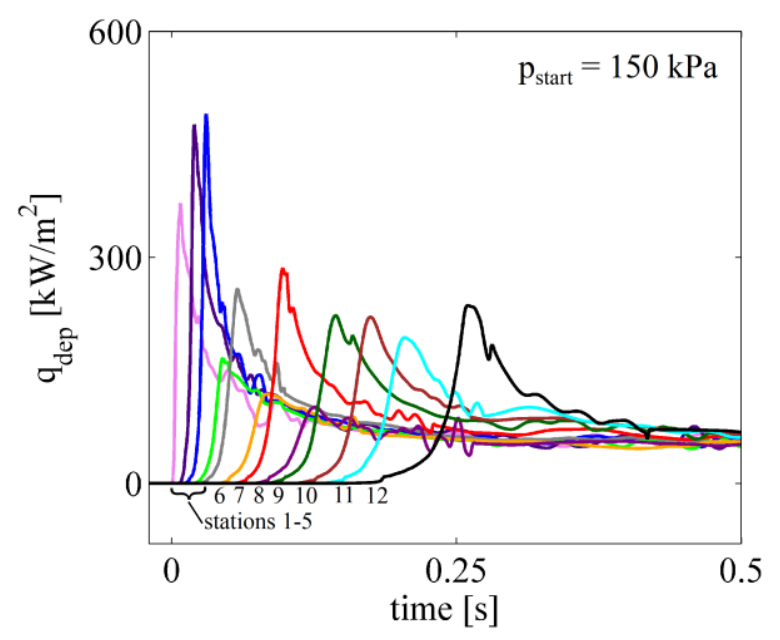

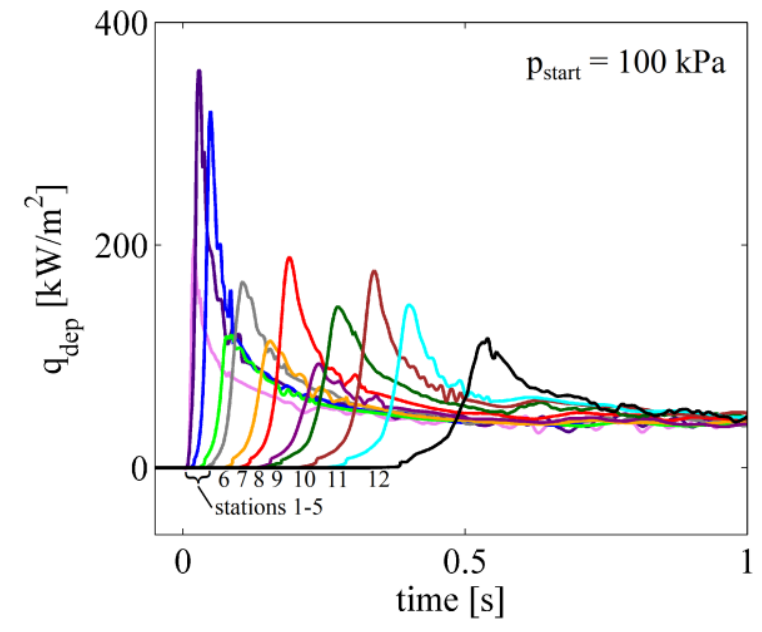

(b)

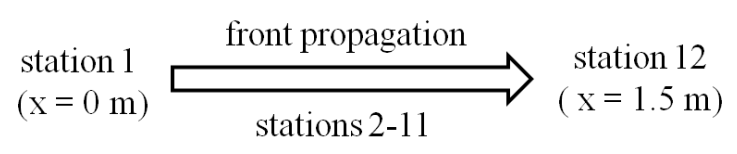

Color key

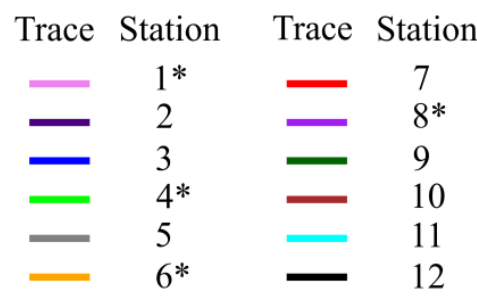

(c)

Figure 5: (Color online) Rate of condensation heat transfer with time at the twelve thermometer stations on the tube during (a) $50 \mathrm{kPa}$ experiment (b) $100 \mathrm{kPa}$ experiment (c) $150 \mathrm{kPa}$ experiment. The gas front travels from station 1 at the entrance of the tube to station 12 near the rigid end. * in the Color key denotes stations with temperature measurement error. 
condensation heat/mass transfer is beyond the scope of present research and so only a conceptual picture has been presented here.

Note that the temperature measurement error at stations 1, 4, 6, and 8 as pointed out in section 4(a) propagates to the condensation heat transfer rates calculated at these stations. As a result of the erroneous temperature traces, the condensation heat transfer traces at these stations are less steep in their rising phase and attain lower than the anticipated peak value.

\section{Results and discussion}

\section{a) Estimation of the mass deposition rate at the front}

To proceed with the analysis of section 3, we now obtain the mass deposition rates from the calculated condensation heat transfer rates and the enthalpy change associated with the condensation process. Recall that equations 4 and 5 use the mass deposition rate 'exactly' at the gas front. Ideally, this deposition rate will exist only at the thin boundary separating the gas and the vacuum and is therefore impossible to measure or to quantify. To avoid this complication we approximate this mass deposition rate at a particular station by the average rate at which the gas deposits at this station in the rising phase of the condensation heat transfer trace. This approximation will indeed yield higher than the 'exact' mass deposition rate, but is based on an observation that is consistent with all the condensation heat transfer traces. Figure 6 illustrates the calculation procedure operating on the condensation heat transfer trace at station 5 of the $150 \mathrm{kPa}$ experiment. The total heat deposited at station 5 from the onset time $t_{\text {onset }}$ to the

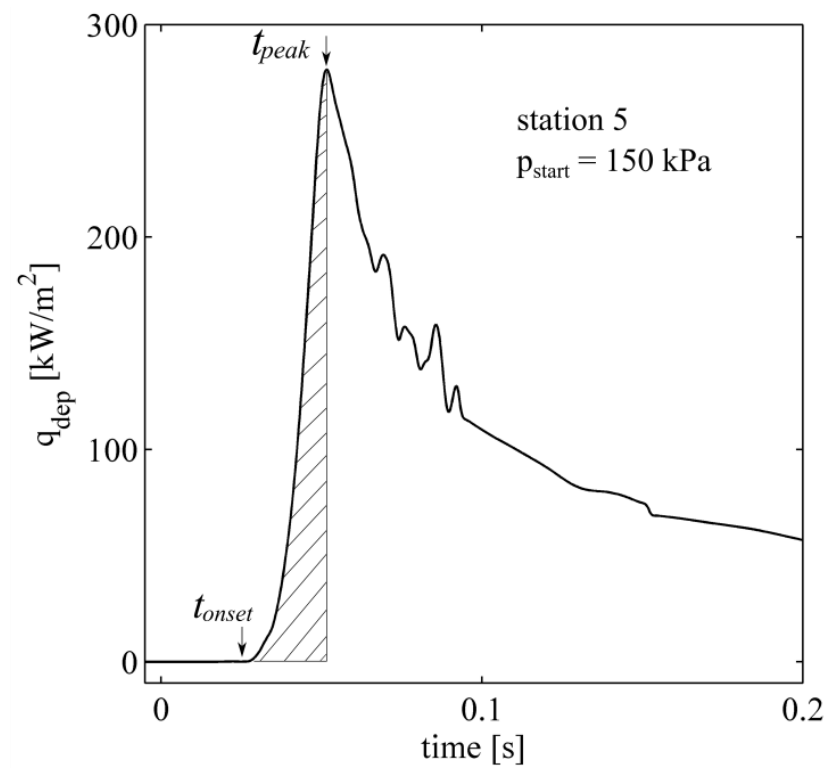

Figure 6: Condensation heat transfer curve for station 5 during $150 \mathrm{kPa}$ experiment: Illustration of the part of the condensation heat transfer curve used to estimate the mass deposition rate at the front. $t_{\text {onset }}$ and $t_{\text {peak }}$ respectively indicate the times of onset and peak condensation heat transfer rates. The shaded area represents the heat deposited at station 5 in the time interval $t_{\text {onset }}$ to $t_{\text {peak }}$. 
time at peak $t_{\text {peak }}$ is the area under the rising phase of the condensation heat transfer curve (the shaded area). The time averaged mass deposition rate in the interval $t_{\text {onset }}$ to $t_{\text {peak }}$ is then:

$$
\overline{\dot{m}}_{\text {dep,front }}^{\prime \prime}=\frac{1}{\Delta h\left(t_{\text {peak }}-t_{\text {onset }}\right)} \int_{t_{\text {onset }}}^{t_{\text {peak }}} q_{\text {dep }}(t) d t
$$

The integral in equation 7 is evaluated using the trapezoid rule. A value of $490 \mathrm{~kJ} / \mathrm{kg}$ for the enthalpy change is used as suggested in [11]. Figure 7 plots $\overline{\dot{m}}_{\text {dep, front }}$ at the twelve stations with their location $x$ relative to the copper tube entrance at $x=0$ (station 1 is at $x=0$ while station 12 is at $x=1.5 \mathrm{~m}$ ).

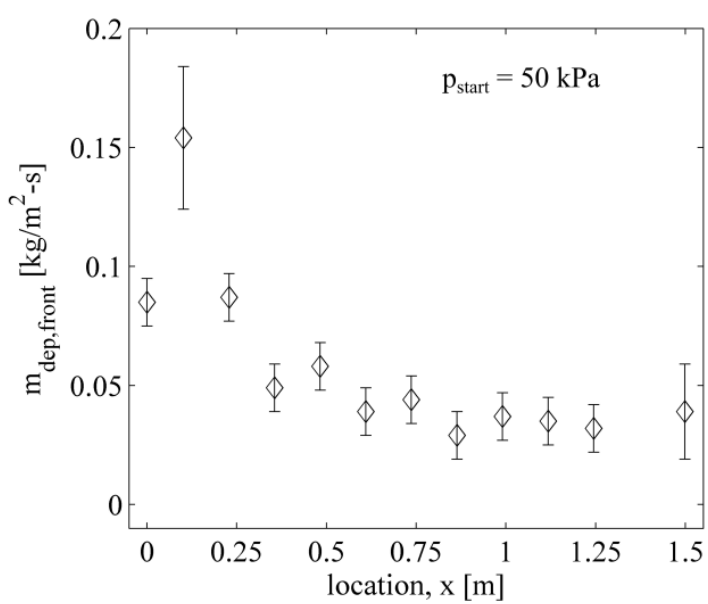

(a)

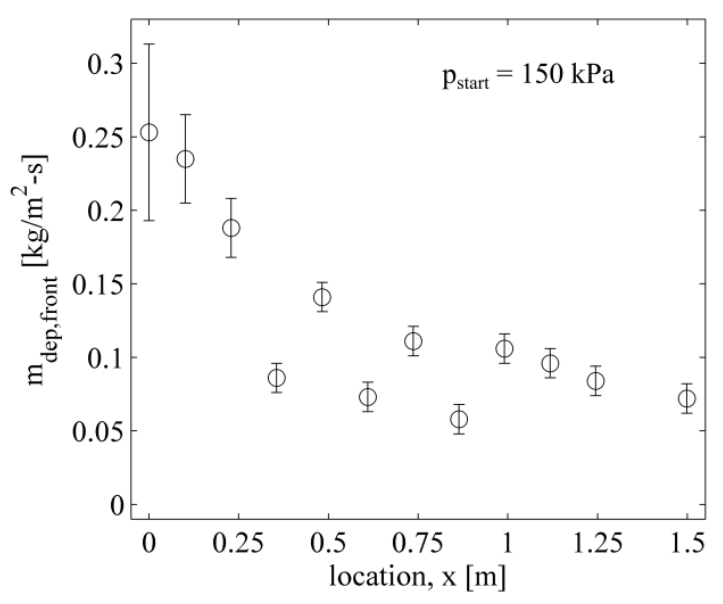

(c)

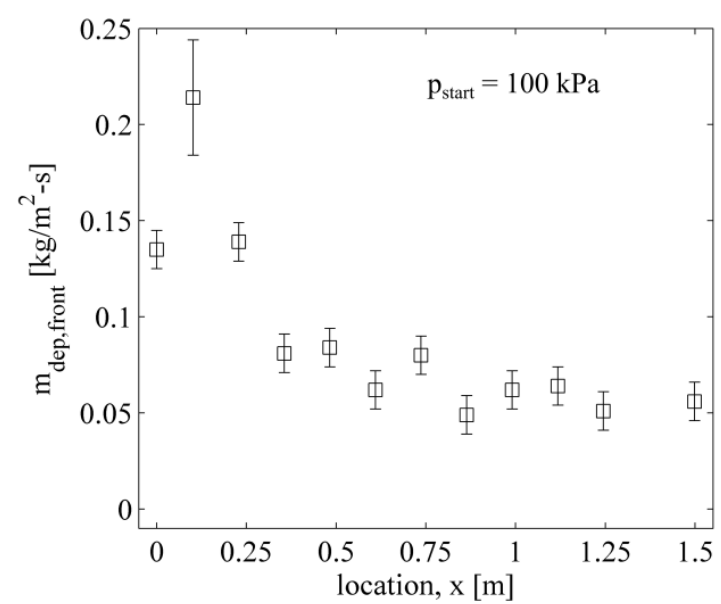

(b)

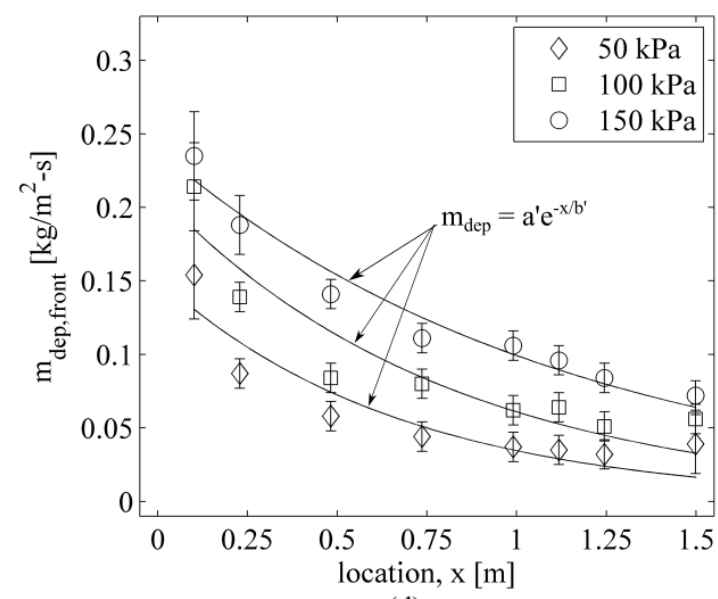

(d)

Figure 7: Mass deposition rates at the twelve stations during (a) $50 \mathrm{kPa}$ experiment (b) $100 \mathrm{kPa}$ experiment (c) $150 \mathrm{kPa}$ experiment while (d) shows the data from the three experiments excluding the outlier points. Exponential curves of the form $\dot{m}_{d e p, \text { front }}^{\prime \prime}=a^{\prime} e^{-x / b^{\prime}}$ are fitted to data in (d). 
The error bars on the data points comprise of the uncertainty introduced by the calculation procedure as well as the temperature measurement uncertainty (see Appendix for uncertainty calculation). The data in figures 7(a), 7(b), and 7(c) are then plotted in figure 7(d) along with exponential fits of the form $\dot{m}_{d e p, \text { front }}^{\prime \prime}=a^{\prime} e^{-x / b^{\prime}}$ to the data set of each experiment. The fit coefficients are calculated by least squares analysis and are tabulated with their standard errors in Table 2. Note that the mass deposition rates at stations $1,4,6$, and 8 are clearly erroneous due to the discrepant temperatures measured at these stations, and so have been excluded from the fits of figure $7(\mathrm{~d})$.

Table 2: Parameters $a^{\prime}$ and $b^{\prime}$ with their standard errors

\begin{tabular}{ccc}
\hline $\mathrm{p}_{\text {start }}[\mathrm{kPa}]$ & $\mathrm{a}[\mathrm{s}]$ & $\mathrm{b}[\mathrm{m}]$ \\
\hline 50 & $0.15 \pm 0.02$ & $0.67 \pm 0.14$ \\
100 & $0.21 \pm 0.02$ & $0.81 \pm 0.14$ \\
150 & $0.24 \pm 0.01$ & $1.14 \pm 0.10$ \\
\hline
\end{tabular}

\section{b) Comparing the exponential decay coefficients}

Finally, we compare in figure 8 the exponential decay coefficient obtained from present analysis to the one determined empirically. The coefficient $b$ was obtained earlier from direction measurement of the propagation speed (see Table 1) and is termed here as 'observation-based'. The coefficient $b$ ' is derived via the reduction of the analytical model (section 3) and the condensation heat transfer analysis. This coefficient is termed as 'analysis-based'. For the three mass in-flow rates vented to the vacuum tube during the $50 \mathrm{kPa}, 100 \mathrm{kPa}$, and $150 \mathrm{kPa}$ experiments, the exponential coefficients obtained from the two independent analyses show fair agreement in trend and in value. The maximum deviation between the two coefficients is $31 \%$ for the $50 \mathrm{kPa}$ experiment. Such deviation is expected because the procedure used to calculate the mass deposition rates at the front is only approximate.

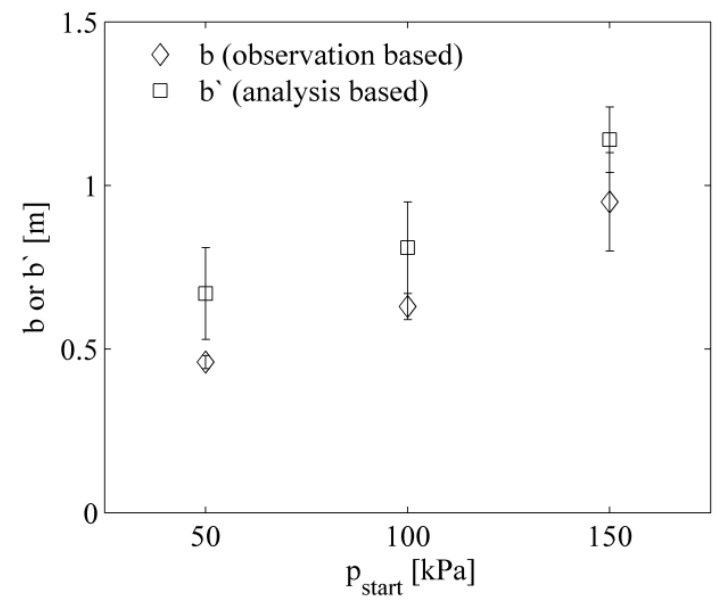

Figure 8: Comparison of the exponential decay coefficients $b$ (based on direct measurement of propagation speed) and $b^{\prime}$ (based on the model and condensation heat transfer analysis) for the three experiments. 


\section{Conclusions}

In this work the propagation of nitrogen gas in a liquid helium cooled tube is analytically studied with the help of the condensation heat transfer rates prevailing during the propagation. Using some assumptions this study puts forward considerable evidence, both analytical and experimental, to support the previously observed exponential decrease in the front speed. Due to inherent difficulties in obtaining a complete analytical solution of the propagation speed [5], the next logical step is to extend this experimental study to tubes of different diameters. This extension will help understand how the empirical parameters $a$ and $b$ vary with the geometry of the vacuum channel and may enable scaling the propagation phenomenon from a laboratory-scale apparatus to real beam-lines.

\section{Acknowledgement}

Work supported by US Department of Energy Grant DE-FG02-96ER40952. We sincerely thank Prof. Wei Guo, Department of Mechanical Engineering and Prof. David Kopriva, Department of Mathematics at Florida State University for their help with the analysis.

\section{Appendix}

A1) Calculation of condensation heat transfer rate [12]

Condensation heat transfer rates at the thermometer stations on the tube are calculated in the following. The heat transfer processes at the tube wall are identified as shown in figure A1.1 and the conservation of energy principle is used to derive an expression for the rate of condensation heat transfer.

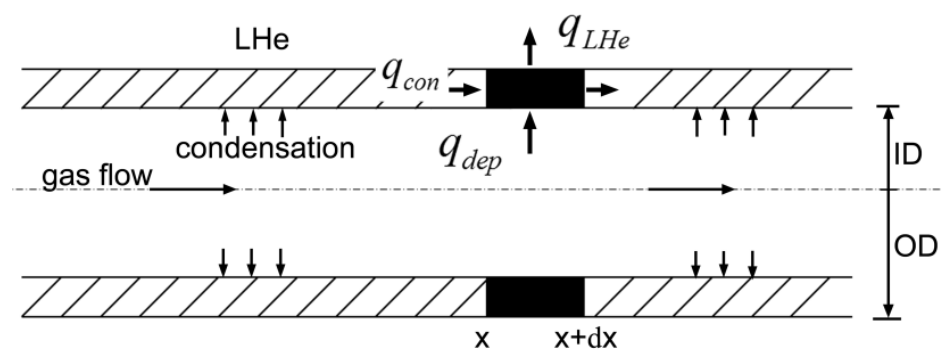

Figure A1.1: Heat transfer processes at the tube wall: $q_{d e p}$ - condensation heat transfer, $q_{c o n}$ - axial heat conduction, and $q_{L H e}$ - heat transfer to LHe.

Conservation of energy (heat balance) yields:

$$
q_{d e p}=A\left(\rho c \frac{d T}{d t}-\frac{d}{d x}\left(k \frac{d T}{d x}\right)\right)+B q_{L H e}
$$

where $\rho$ is the density of the tube material $\left(8960 \mathrm{~kg} / \mathrm{m}^{3}\right.$ for copper) while $c$ and $k$ respectively are the temperature dependent specific heat capacity and thermal conductivity (available for copper at [13]). $A=O D^{2}-I D^{2} / 4 I D$ and $B=O D / I D$ are geometrical constants of the tube. The two terms in the first bracket on the RHS are the rate of heat accumulation in tube and the gradient of conduction heat 
transfer rate. The condensation heat transfer rate $q_{d e p}$ can be calculated by evaluating the RHS terms individually in the following way:

a) Rate of heat accumulation in the tube, $A \rho c(d T / d t)$

The raw temperature data contains random noise and is smoothed prior to calculating the time rate of change. Smoothing is necessary because evaluating the time derivative from raw data simply amplifies the noise and renders the derivative useless. We have used two smoothing methods- piecewise smoothing splines and Savitzky-Golay [14]. Reasons for using two different methods are stated in Section B of this appendix where we also compare the outcomes. The rate of heat accumulation calculated using spline smoothed temperature is displayed in figure A1.4(a).

b) Gradient of conduction heat transfer rate, $A d(k d T / d x) / d x$

The calculation of $A d(k d T / d x) / d x$ at a thermometer station proceeds by discretizing this double derivative around that station. Consider, as shown in figure A1.2, a 1-D three-node grid centered at $x$ and with node spacing $\Delta x$. The central node of this grid aligns with a thermometer, say at station 5 , so the temperature vs. time trace at this node is available. The double derivative will discretize on this grid as:

$$
\left.A \frac{d}{d x}\left(k \frac{d T}{d x}\right)\right|_{x}=A \frac{k_{x-\Delta x / 2}\left(T_{x}-T_{x-\Delta x}\right)-k_{x+\Delta x / 2}\left(T_{x+\Delta x}-T_{x}\right)}{\Delta x^{2}}
$$
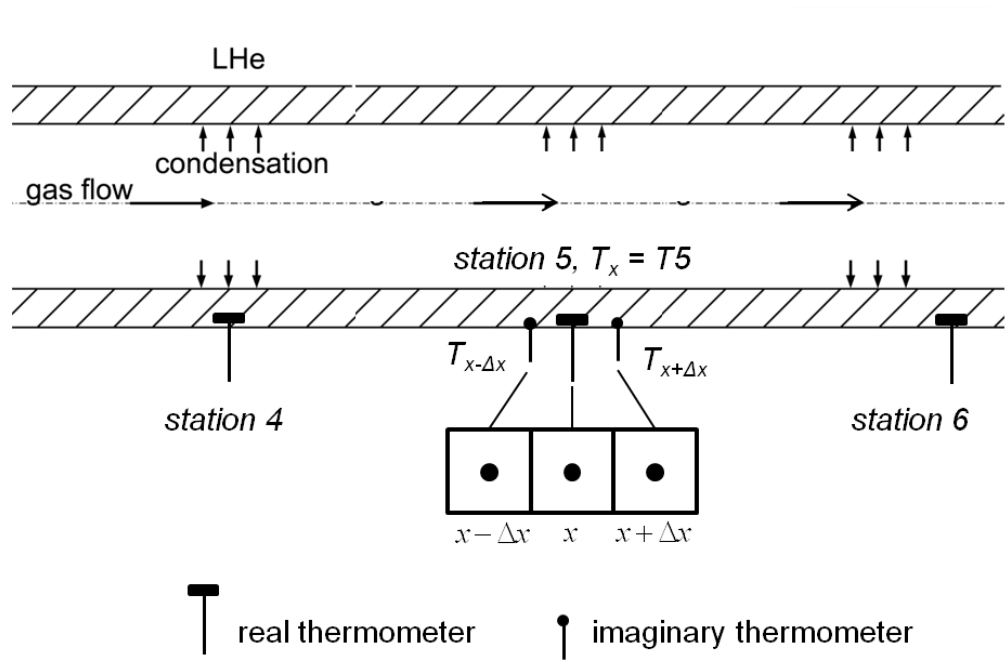

Figure A1.2: An illustration of the method used to generate temperature profiles from the measured temperature data.

Evaluating $A d(k d T / d x) / d x$ using equation A1.2 requires $\Delta x$ to be small enough so that the thermal conductivity $k$ is uniform over $\Delta x$. The temperature data $T_{x-\Delta x}$ and $T_{x+\Delta x}$ required for evaluating equation A1.2 are assumed to come from two thermometers that are imagined to be $\Delta x$ upstream and $\Delta x$ downstream of station 5 (see figure A1.2). Note that these data are not real measurements. They are 
generated using the wave propagation principle by assuming the temperature profiles $T(t)$ at $x-\Delta x$ and at $x+\Delta x$ to have the same shape as that of the profile measured at $x$ (station 5). Mathematically, the three temperature profiles can then be expressed as:

$$
\begin{aligned}
& T_{x-\Delta x}(t)=T_{x}(t+\Delta t) \\
& T_{x}(t)=T_{x}(t) \\
& T_{x+\Delta x}(t)=T_{x}(t-\Delta t)
\end{aligned}
$$

In equations $\mathrm{A} 1.3$ and $\mathrm{A} 1.5, \Delta t$ is the time taken by the temperature profile to propagate from $x-\Delta x$ to $x$ (or from $x$ to $x+\Delta x$ ). Equivalently, $\Delta x / \Delta t$ is the propagation speed. The smallest timescale in our experiment that can be used as $\Delta t$ is the sampling interval of a thermometer $(0.21 \mathrm{~ms}$, sampling rate is $4.8 \mathrm{kHz})$. Figure A1.3 shows the measured temperature profile $T_{x}(t)$ at station 5 and the generated temperature profiles $T_{x-\Delta x}$ and $T_{x+\Delta x}$ using $\Delta t=0.21 \mathrm{~ms}$. Clearly $T_{x+\Delta x}(t)-T_{x}(t)$ or $T_{x}(t)-T_{x-\Delta x}(t)$, i.e., the temperature difference over the node spacing is not large $(<100 \mathrm{mK})$ and so $k$ can be taken to be uniform over $\Delta x$. Thermal conductivity for the region between two adjacent nodes is then calculated at the mean temperature of the nodes. Next, $\Delta x$ is obtained by multiplying the propagation speed $\Delta x / \Delta t$ with the propagation time $\Delta t$. The propagation speed over the section $x-\Delta x$ to $x+\Delta x$ is taken to be the propagation speed at $x$, which from direction measurement at this station has been determined to be $16 \mathrm{~m} / \mathrm{s}$ (obtained from the data in Table 1). $\Delta x$ is then $\approx 3 \mathrm{~mm}$, which is small enough so as to support our assumption of the temperature profile not changing shape considerably between $x-\Delta x$ and $x+\Delta x$.

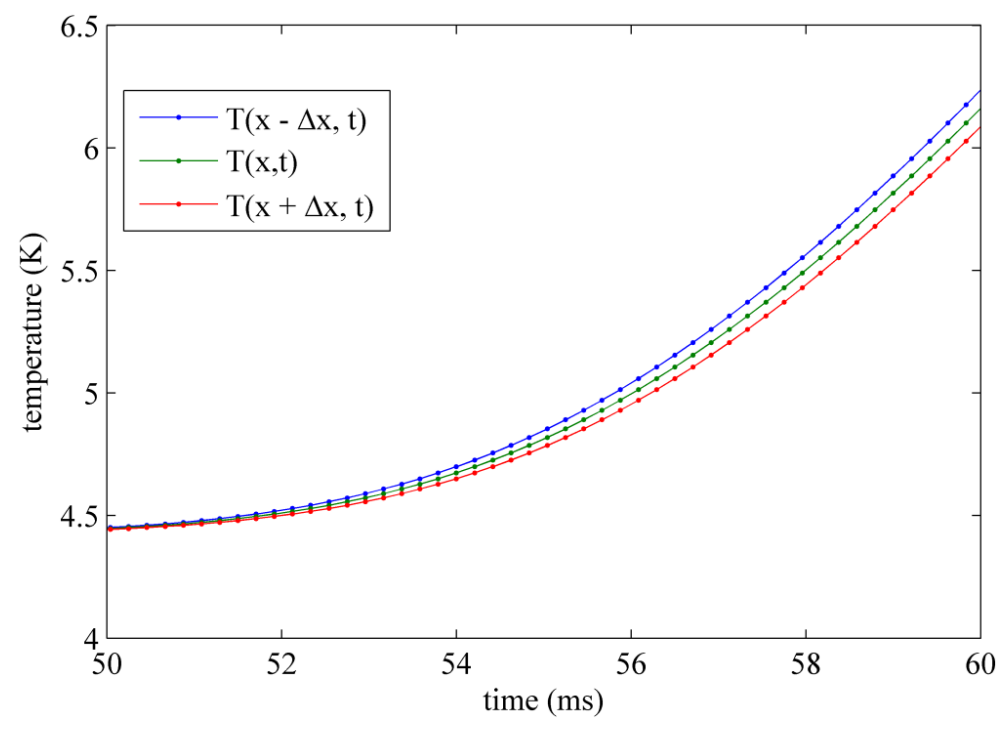

Figure A1.3: (Color online) Comparing the generated temperature profiles with the measured one. The comparison is shown for $10 \mathrm{~ms}$ but the form is consistent for the entire duration of the measurements.

The derivative of heat conduction $A d(k d T / d x) / d x$ is then evaluated using equation A1.2. The result of this procedure is displayed in figure A1.4(b). 


\section{c) LHe heat transfer rate, $B q_{\text {LHe }}$}

The heat transfer rates to LHe in our experiment are not explicitly measured, but are estimated from the tube temperature data and the known correlations and/or heat transfer data from literature. The calculated LHe heat transfer rates correspond to the steady LHe heat fluxes that would originate from a tube at the temperature measured during experiments. Clearly for time $<0.5 \mathrm{~s}$, the tube temperature is unsteady and so the heat transfer rates obtained using steady state correlations/data only approximate the actual LHe heat transfer rates that exist in our experiments. The LHe heat transfer rate in the observed nucleate boiling regime $(\mathrm{T}<4.56 \mathrm{~K})$ is estimated using the simplified Kutateladze correlation for a saturated pool

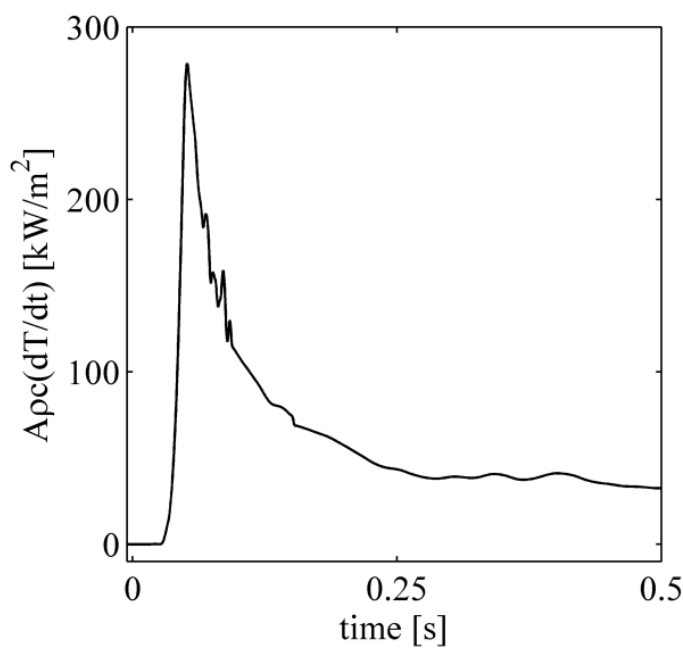

(a)

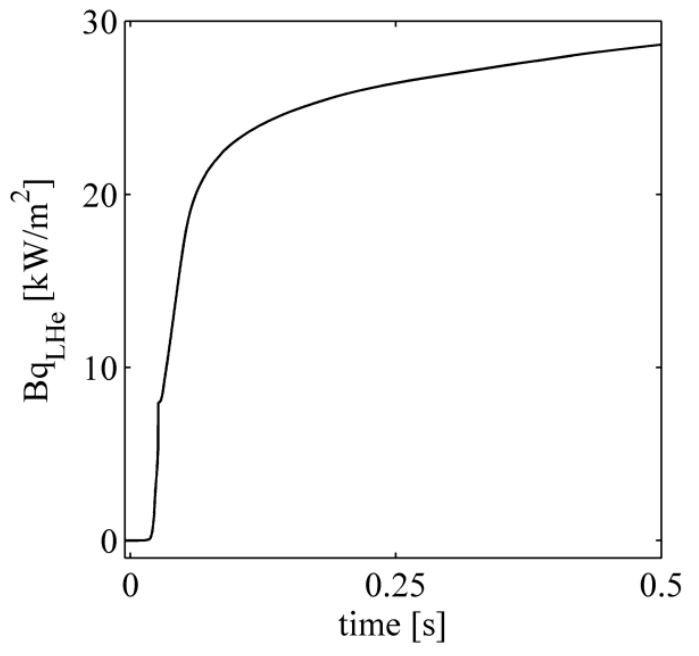

(c)

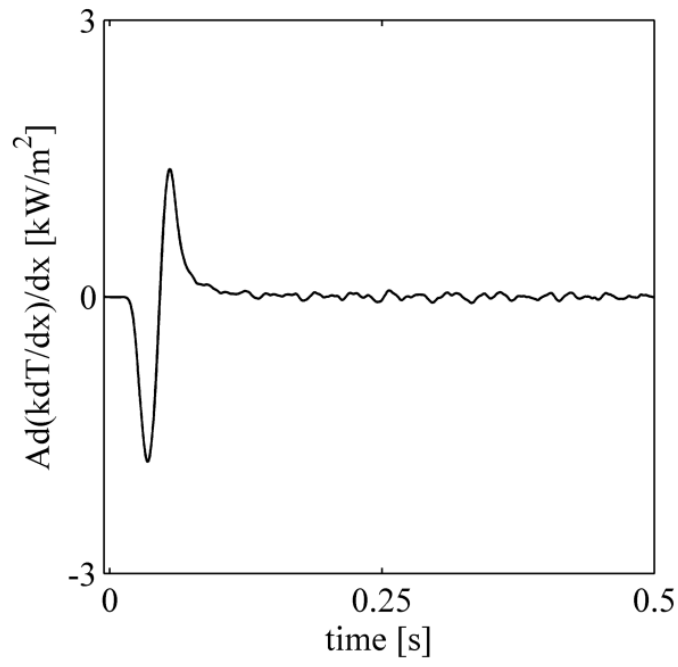

(b)

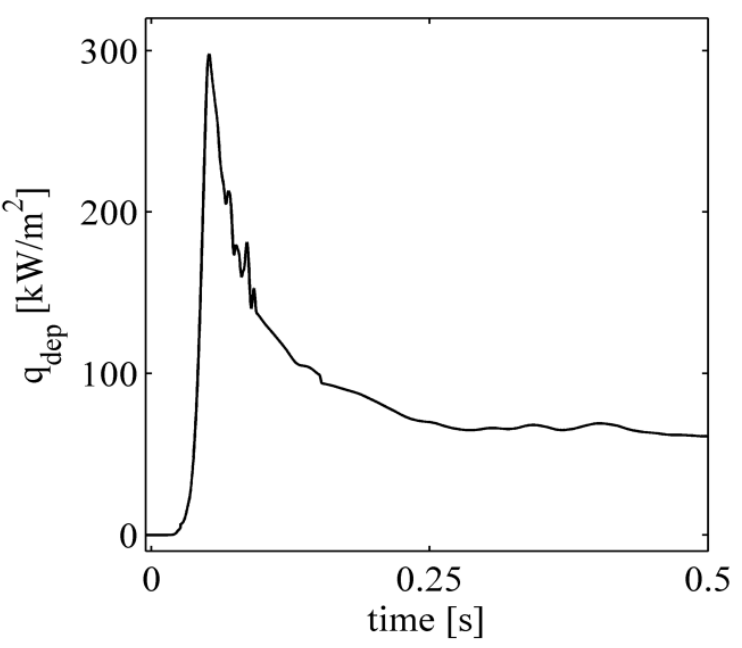

(d)

Figure A1.4: Calculated heat transfer modes appearing in equation A1.1 (a) rate of change of energy content of the tube (b) spatial derivative of axial conduction (c) LHe heat transfer rate (d) condensation heat transfer or heat deposition rate. 
of liquid helium at $4.2 \mathrm{~K}[1]$ :

$$
q_{L H e}\left[k W / m^{2}\right]=58(T-4.2)^{2.5}
$$

In the film boiling range ( $\mathrm{T}>4.56 \mathrm{~K}$ ), the heat transfer rates are estimated using the data of Steward [7]. Assuming a constant film boiling heat transfer coefficient, the steady state LHe heat transfer rate measured by Steward can be represented by the relation:

$$
q_{L H e}\left[k W / m^{2}\right]=0.423(T-4.2)+6.495
$$

The calculated LHe heat transfer rate at station 5 is shown in figure A1.4(c). Adding the individual heat transfer modes in equation A1.1 yields the condensation heat transfer rate at station 5 . The rate is plotted with time in figure A1.4(d). Note that the derivative of axial conduction has negligible value compared to the rate of heat accumulation in the tube as well as to the LHe heat transfer rate, so that this mode can be dropped from the heat balance. Equation A1.1 then simplifies to:

$$
q_{d e p}=A \rho c \frac{d T}{d t}+B q_{L H e}
$$

A2) Uncertainty calculation

The uncertainty in temperature comprising of calibration error and random noise is $20 \mathrm{mK}$ below $20 \mathrm{~K}$ and $0.2 \mathrm{~K}$ above $20 \mathrm{~K}$. This uncertainty propagates to the instantaneous condensation heat transfer rate via the heat balance equation A1.8. The maximum uncertainty in $q_{d e p}$ is then:

$$
\begin{aligned}
\delta q_{\text {dep }} & =A \rho\left|\delta\left(c \frac{d T}{d t}\right)\right|+B\left|\delta q_{L H e}\right| \\
& =A \rho\left|\frac{d T}{d t} \delta c+c \delta\left(\frac{d T}{d t}\right)\right|+B\left|\delta q_{L H e}\right|
\end{aligned}
$$

a) Uncertainty in specific heat capacity, $\delta c$

The temperature dependence of specific heat of copper is given by the empirical fit [13]:

$$
c=10^{y}, y=\sum_{i=0}^{7} a_{i}(\log T)^{i}
$$

where $\log$ is to base 10 and the coefficients $a_{i}$ are summarized in Table A2.1. As the coefficients in Table A2.1 are given to be accurate to more than $99.99 \%$ we neglect the uncertainty in specific heat due to the uncertainties in these coefficients. The uncertainty in specific heat capacity will then be $\delta c=(d c / d T) \delta T$. The maximum $\delta c$ is $0.065 \mathrm{~J} / \mathrm{kg}-\mathrm{K}$ below and $0.65 \mathrm{~J} / \mathrm{kg}-\mathrm{K}$ above $20 \mathrm{~K}$. 
Table A2.1: Coefficients in the specific heat vs. temperature fit (equation A2.2) [13]

\begin{tabular}{cccc}
\hline Coefficient & Value & Coefficient & Value \\
\hline$a_{0}$ & -1.91844 & $a_{4}$ & 21.9661 \\
$a_{1}$ & -0.15973 & $a_{5}$ & -12.7328 \\
$a_{2}$ & 8.61013 & $a_{6}$ & 3.54322 \\
$a_{3}$ & -18.996 & $a_{7}$ & -0.3797 \\
\hline
\end{tabular}

b) Uncertainty in LHe heat transfer rate, $\delta q_{L H e}$

The maximum $\delta q_{L H e}$ in the nucleate boiling regime is $600 \mathrm{~W} / \mathrm{m}^{2}$. In the film boiling range maximum $\delta q_{L H e}$ is $10 \mathrm{~W} / \mathrm{m}^{2}$ below $20 \mathrm{~K}$ and $100 \mathrm{~W} / \mathrm{m}^{2}$ above $20 \mathrm{~K}$. These uncertainties are based only on the tube temperature uncertainties and do not account for the error that the steady state correlations/data would introduce in calculating the unsteady rates.

c) Uncertainty in the rate of change of temperature, $\delta(d T / d t)$

Uncertainty in the time rate of change of temperature sources from the temperature smoothing procedure. We have used two methods to generate smooth temperature data. The first method uses piecewise smoothing splines, which provide an excellent mean temperature but over-complicate estimating the uncertainty in the derivative with time. The second method is to fit polynomials to the raw data, again in a piecewise manner, using the simplified least squared procedures devised by Savitzky and Golay [14]. As illustrated below, this procedure allows one to conveniently determine the smoothed mean temperature, its time rate of change $d T / d t$, and also the uncertainty $\delta(d T / d t)$.

The Savitzky-Golay procedure fits a polynomial of degree $m$ to the data contained in a window of size $2 w+1$. Of interest in operating over a particular window is to determine the smooth temperature, the derivative, and the derivative uncertainty only at the center of this window. The window is then moved across the data so that the quantities of interest may be calculated at every data point. In our analysis, we have used $m=2$ and $w=10$. Consider the procedure operating on a window $j$ centered at data point at $t_{j}$. In this case, the fitting polynomial is:

$$
T_{j}=b_{0, j}+b_{1, j} \bar{t}+b_{2, j} \bar{t}^{2}
$$

where $\bar{t}=t-t_{j} / \Delta t$, i.e., the abscissa of a data-point in the window relative to the abscissa of the window center-point $t_{j}$, normalized by the spacing $\Delta t$ between adjacent data points. In simple terms, $\bar{t}$ represents the abscissa-distance of a data-point at $t$ in a window from the center of that window at $t_{j}$ in units of the spacing $\Delta t$. With this transformation $b_{0, j}, b_{1, j} / \Delta t$, and $\delta b_{1, j} / \Delta t$ respectively are the smooth temperature, the temperature derivative with time, and the uncertainty in derivative of temperature at $t_{j}$ (window center). The fit parameters for the window $j$ are given by: 


$$
\begin{aligned}
& b_{0, j}=\sum_{i=-w}^{w} C_{0 i, j} T_{i, j} \\
& b_{1, j}=\sum_{i=-w}^{w} C_{1 i, j} T_{i, j} \\
& b_{2, j}=\sum_{i=-w}^{w} C_{2 i, j} T_{i, j}
\end{aligned}
$$

In these expressions, the coefficients $C_{0 i, j}, C_{1 i, j}$, and $C_{2 i, j}$ are obtained by the procedure described in [14]. The fit uncertainty [15] for window $j$ is then:

$$
\delta_{j}=\sqrt{\frac{1}{2 w-1} \sum_{i=-w}^{w}\left(T_{i, j}-T_{j}\left(t_{i}\right)\right)^{2}}
$$

which when propagated through the expression for $b_{1, j}$ (equation A2.4) yields $\delta b_{1, j}$.

This procedure is extended to find the time rate of change of temperature, $b_{1} / \Delta t$ and the uncertainty in this rate, $\delta(d T / d t)=\delta b_{1} / \Delta t$ at every point in the temperature-time data set. Figure A2.1 shows the result of a sample calculation. Equation A2.1 then yields $\delta q_{d e p}$.

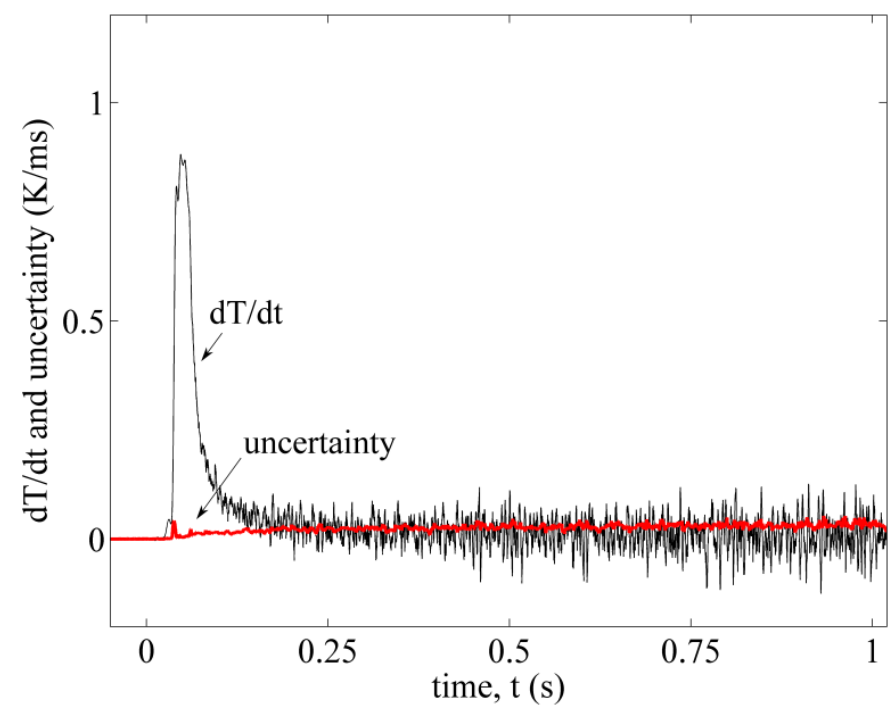

Figure A2.1: (Color online) Time rate of change of temperature and uncertainty calculated using SavitzyGolay procedure.

The maximum error in the mass deposition rate at the front, according to its definition, is: 


$$
\delta \dot{m}_{d e p}^{\prime \prime}=\frac{1}{n \Delta h} \sum_{n}\left|\delta q_{d e p, i}\right|
$$

where $n$ is the number of time steps from the onset of condensation till the condensation rate becomes maximum. Note that $\delta(d T / d t)$ and consequently $\delta \dot{m}_{d e p}$ will change if different $m$ and $w$ are used and so the uncertainty can be minimized by choosing optimal $m$ and $w$. We, however, have not attempted to optimize these parameters.

We finally compare in figure A2.2 the condensation heat transfer rates calculated using smoothing splines and using Savitzky-Golay smoothing procedure. This comparison shows that both techniques yield same condensation heat transfer rate in its rising phase and so can be used interchangeably to calculate $\dot{m}_{d e p}$ near the front. The uncertainty is calculated using the Savitzky-Golay procedure.

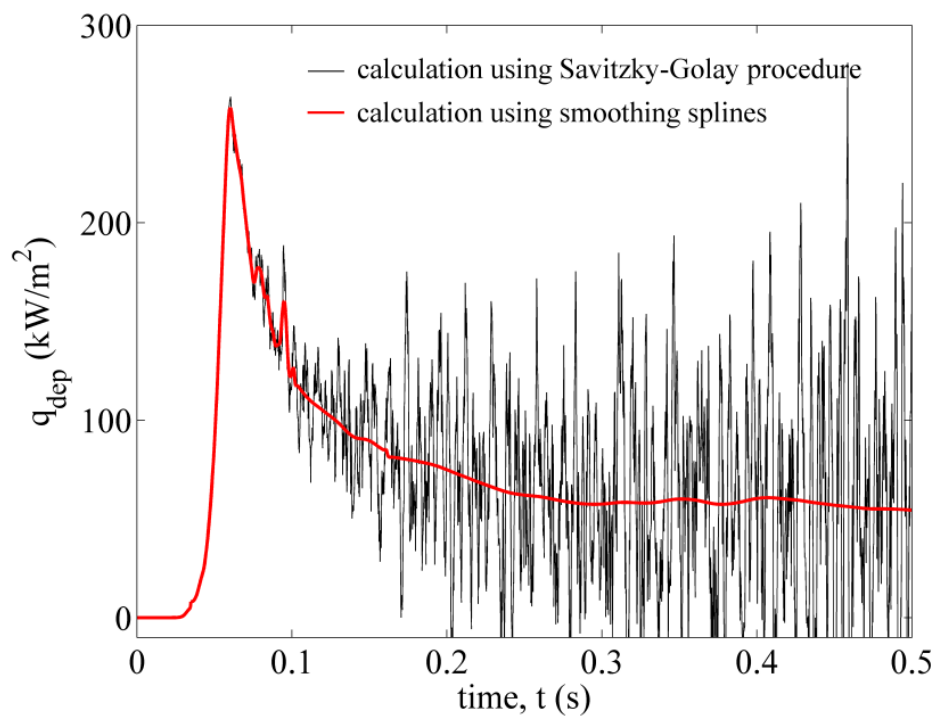

Figure A2.2: (Color online) Comparison of condensation heat transfer rates calculated by smoothing the raw temperature using smoothing splines and using Savitzky-Golay procedure.

\section{References}

[1] S. W. Van Sciver, "Helium Cryogenics", $2^{\text {nd }}$ ed., Springer-Verlag, (2012), p. 247.

[2] T. Boeckman et al., "Experimental Tests of Fault Conditions during the Cryogenic Operation of a XFEL Prototype Cryomodule," Proceedings of the International Cryogenic Engineering Conference 22 International Cryogenic Materials Conference- 2008, Seoul (2009).

[3] A. A. Dalesandro, R. C. Dhuley, J. C. Theilacker, and S. W. Van Sciver, "Results from sudden loss of vacuum on scaled superconducting radio frequency cryomodule experiment," AIP Conference Proceedings, 1573, pp. 1822-1828, (2014). http://dx.doi.org/10.1063/1.4860929 
[4] T. Takiya, F. Higashino, Y. Terada, and A. Komura, "Pressure wave propagation by gas expansion in a high vacuum tube," Journal of Vacuum Science and Technology- A, 17, pp. 2059-2063, (1999). http://dx.doi.org/10.1116/1.581726

[5] R. C. Dhuley and S. W. Van Sciver, "Experimental and analytical studies of nitrogen gas propagation in a liquid helium cooled vacuum tube following sudden vacuum loss," International Journal of Heat and Mass Transfer, (under review).

[6] R. C. Dhuley and S. W. Van Sciver, "Sudden Vacuum Loss in Long Liquid Helium Cooled Tubes," IEEE Transactions on Applied Superconductivity, 25(3), Article\# 9000305, (2015). http://dx.doi.org/10.1109/TASC.2014.2367156

[7] W. G. Steward, “Transient helium heat transfer phase I-Static coolant," International Journal of Heat and Mass Transfer, 21(7), pp. 863-874, (1978). http://dx.doi.org/ 10.1016/0017-9310(78)90178-3

[8] C. Tzemos, M. Gegenheimer, and C. B. Hood, Jr, "Design and performance of a liquid helium cryopump operating in the viscous flow regime," Advances in Cryogenic Engineering, 31, pp. 575-581, (1986). http://dx.doi.org/10.1007/978-1-4613-2213-9 66

[9] G. Davey, "Cryopumping in the transitional and continuum pressure regions," Vacuum, 26(1), pp. 1722, (1975). http://dx.doi.org/10.1016/S0042-207X(76)80414-9

[10] M. E. Bland, "The cryopumping of water vapour in the continuum pressure region," Cryogenics, 15(11), pp. 639-643, (1975). http://dx.doi.org/10.1016/0011-2275(75)90094-6

[11] R. B. Scott, “Cryogenic Engineering”, 3rd ed., Met-Chem Research Inc., (1988), p. 279.

[12] R. C. Dhuley and S. W. Van Sciver, "Heat transfer in a liquid helium cooled vacuum tube following sudden vacuum loss," IOP Conference Series: Material Science and Engineering, (accepted for publication).

[13] NIST Cryogenic Material Properties Database, properties of copper available at http://www.cryogenics.nist.gov/

[14] A. Savitzky and M. J. E. Golay, "Smoothing and differentiation of data by Simplified Least Squares Procedures," Analytical Chemistry, 36(8), pp. 1627-1639, (1964).

http://dx.doi.org/10.1021/ac60214a047.

[15] J. R. Taylor, "An introduction to error analysis: The study of uncertainties in physical measurements," $2^{\text {nd }} e d .$, University Science Books, (1997), p. 187. 